\title{
X-ray-standing-wave interface studies of germanium on Sil111)
}

\section{Citation}

Patel, J. R., J. A. Golovchenko, J. C. Bean, and R. J. Morris. 1985. X-Ray-Standing-Wave Interface Studies of Germanium on Si(111). Physical Review B 31, no. 10: 6884-6886. doi:10.1103/ physrevb.31.6884.

\section{Published Version}

doi:10.1103/PhysRevB.31.6884

\section{Permanent link}

http://nrs.harvard.edu/urn-3:HUL.InstRepos:29407047

\section{Terms of Use}

This article was downloaded from Harvard University's DASH repository, and is made available under the terms and conditions applicable to Other Posted Material, as set forth at http:// nrs.harvard.edu/urn-3:HUL.InstRepos:dash.current.terms-of-use\#LAA

\section{Share Your Story}

The Harvard community has made this article openly available.

Please share how this access benefits you. Submit a story.

\section{Accessibility}




\title{
$\mathrm{X}$-ray-standing-wave interface studies of germanium on $\mathrm{Si}(111)$
}

\author{
J. R. Patel, J. A. Golovchenko, J. C. Bean, and R. J. Morris* \\ AT\&T Bell Laboratories, Murray Hill, New Jersey 07974 \\ (Received 28 January 1985)
}

\begin{abstract}
The position of germanium atoms on a $\mathrm{Si}(111)(7 \times 7)$ surface prepared in a molecular-beam-epitaxy system has been established using the characteristic fluorescence generated by $\mathrm{x}$-ray standing waves. For submonolayer coverages germanium occupies both types of (111) lattice sites. Measurements of the germanium position relative to planes inclined to the surface do not support recent stacking-fault models for the $\mathrm{Si}(111)(7 \times 7)$ surface.
\end{abstract}

Recently there has been a growth of interest in phenomena associated with crystal interfaces. In particular, several advances have been made in the experimental methods of probing crystal surfaces and interfaces. Of fundamental importance in the understanding of such boundaries is a knowledge of the geometrical surface structure, which often is not a simple termination of the bulk crystal. ${ }^{1}$ For instance, an annealed $\mathrm{Si}(111)$ surface prepared in an ultrahigh-vacuum (UHV) environment adopts a superlattice structure with 49 atoms in the surface unit cell. This detailed structure is still unexplained though it is actively being studied by a variety of methods including low-energy electron diffraction (LEED),${ }^{1}$ ion scattering, ${ }^{2}$ electron microscopy, ${ }^{3}$ tunneling microscopy,${ }^{4}$ and $\mathrm{He}$ atom scattering. ${ }^{5}$ An interesting extension of these studies has involved the investigation of surface impurity atoms on well characterized surfaces. A particularly fascinating case of this kind is germanium adsorbed on the previously mentioned silicon surface. Although several investigators have observed the influence of adsorbed germanium layers on surface structure ${ }^{6-8}$ none has provided concrete results on the actual position of the germanium atoms relative to the crystal below. In view of the chemical similarity between $\mathrm{Ge}$ and $\mathrm{Si}$, such information should provide a valuable guide to understanding the atomic structure of the interface and the method by which it advances during crystal growth.

Atom-location studies using $\mathrm{x}$-ray standing waves have recently demonstrated the ability to locate surface impurity atoms and their registration relative to the crystal below for the case of bromine atoms ${ }^{9}$ on $\mathrm{Si}(111)$. Owing to the relatively simple manner in which the bromine silicon bond is capable of terminating the crystal lattice, straightforward results with bromine atoms occupying a unique lattice site above the (111) double layer were demonstrated. ${ }^{9}$ For the case of less than one monolayer (ML) of germanium on silicon, the results would seem more difficult to anticipate. For example, the atoms may be envisioned as occupying either or both of two distinct atomic planes which might be associated with the topmost atomic layer at the surface. Recent standing-wave experiments on GaAs (Ref. 10) have demonstrated the ability to distinguish between these two kinds of sites for atoms in the bulk. Hence the application of the standing-wave method to the $\mathrm{Ge}$ on $\mathrm{Si}$ problem seems particularly timely, and as we shall show, provides significantly new information about the interface.

Germanium atoms were evaporated onto silicon samples in a molecular-beam-epitaxy (MBE) system. ${ }^{11}$ The (111) surface was sputter cleaned with argon atoms and annealed to $800^{\circ} \mathrm{C}$ after which a silicon epitaxial layer $2000 \AA$ thick was grown at $750^{\circ} \mathrm{C}$. The $(7 \times 7)$ surface diffraction structure was then observed by LEED on the epitaxial surface layer. Care in mounting and uniform heating of our 4mm-thick samples was necessary to avoid plastic deformation which would broaden the narrow natural $\mathrm{x}$-ray diffraction widths and hence complicate the interpretation of standing-wave lattice-location information. The sample was then cooled to $\sim 300^{\circ} \mathrm{C}$ and $0.8 \mathrm{ML}$ of germanium was deposited on the surface at a deposition rate of $1 \mathrm{ML} / \mathrm{sec}$. LEED experiments showed no diminution of the $(7 \times 7)$ electron diffraction pattern. The sample was then cooled to room temperature under high vacuum and a $100 \AA$ protective cap of amorphous silicon was deposited as the final preparation step so as to "freeze in" the surface morphology and prevent any surface reactions with the atmosphere in subsequent handling and standing-wave experiments.

The sample was withdrawn from the MBE apparatus and studied by Rutherford backscattering and channeling to verify the amorphous nature of the cap as well as the germanium surface coverage. Absolutely no germanium dip was observed during (111) channeling in the bulk, which implies that the silicon above the germanium is noncrystalline and no germanium diffusion into bulk crystalline sites has occurred. ${ }^{12}$

The $300^{\circ} \mathrm{C}$ deposition temperature was chosen based on the Auger electron spectroscopy (AES) work of Gossmann, Feldman, and Gibson ${ }^{8}$ such that no germanium in-diffusion would occur as was verified by the channeling. Earlier AES and channeling studies had suggested that growth proceeded in a layer by layer mode.6,7 As we shall see, this is not supported by the standing-wave experiment.

The capped MBE crystal was then exposed to the ambient atmosphere in which standing-wave experiments were performed. The results for crystal reflectivity and fluorescence are shown in Fig. 1. The modulation of the germanium fluorescence in the total reflection region reflects the position of the germanium atoms on the surface as the exciting $\mathrm{x}$-ray standing-wave phase is translated perpendicular to the (111) planes. The inset in Fig. 1 contains a model of the silicon lattice (111) planes, and it will be noted that substitutional germanium surface atoms might occupy either $A$ or $B$ plane sites depending on how the surface terminates. The theoretical curves $A$ and $B$ shown in Fig. 1 illustrate the expected angular dependence of germanium fluorescence for those two cases. The data shown in Fig. 1 clearly follow neither of these two curves.

A model-independent method of stating our results is that within $0.06 \AA$ the maximum of the (111) Fourier component of the germanium charge density lies directly 


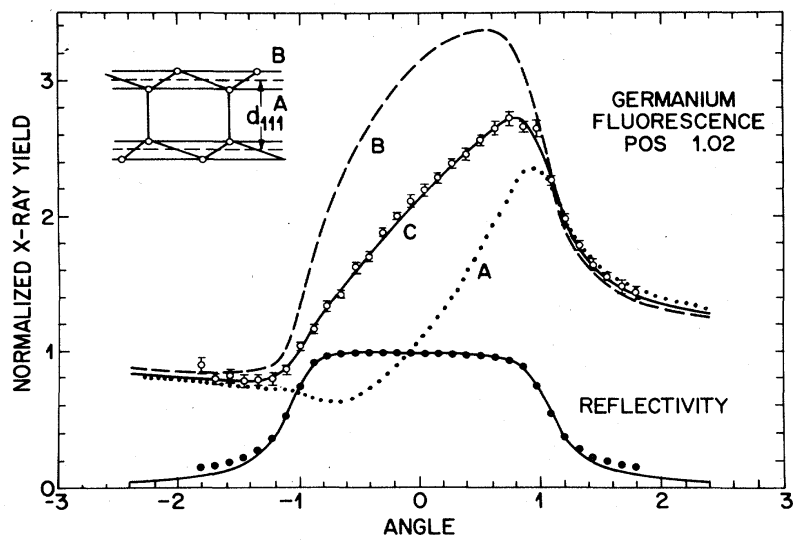

FIG. 1. Germanium fluorescence and reflectivity angular yields for (111) Bragg diffraction on a $\mathrm{Si}(111)$ surface. Curves $\mathrm{A}$ and $\mathrm{B}$ represent theoretical fluorescence yields if germanium occupied sites $A$ and $B$, respectively. (see inset). Curve $\mathrm{C}$, theory and experiment corresponding to germanium occupying both $A$ and $B$ sites.

between the surface close-spaced silicon (111) planes. A density distribution having equal numbers of germanium atoms on the topmost $A$ and $B$ planes is the most straightforward explanation of this result. This assignment is corroborated by the results obtained from a (220) flurorescence study discussed below. Essentially identical results have been obtained for coverages of germanium as low as 0.2 ML. The absence of preference for either $A$ or $B$ type sites suggests that layer by layer growth proposed in several earlier studies does not occur. In terms of this assignment it is interesting to note that most of the germanium atoms are located coherently on the surface with the small fraction occupying unknown random positions. We now emphasize that the information gained so far deals only with the germanium density distribution perpendicular to the (111) planes since these were the diffracting planes. As we have shown ${ }^{9}$ previously, further information on atom positions along other directions can be obtained by considering standing-wave effects for reflections whose repicrocal lattice vectors are not normal to the surface. We have studied the reflection from (220) planes which lie at $35.26^{\circ}$ to the (111) surface because, as we shall see, data for these directions bear directly on stacking-fault models for explaining reconstructed silicon surfaces. ${ }^{13-15}$

The principle of the method can best be demonstrated with the aid of [110] projection of the diamond structure. Shown in Fig. 2 is a schematic diagram of the [110] projection showing (111) planes parallel to the surface. The right-hand part of the surface plane is shown in the stacking-fault configuration, where the regular stacking sequence $A A^{\prime} B B^{\prime} C C^{\prime} A A^{\prime}$ is modified by the fault to $A A^{\prime} B B^{\prime} C C^{\prime} B B^{\prime}$. We can now imagine that the surface is terminated by an impurity atom similar to the host. For the silicon lattice the chemically similar germanium impurity at the surface would be an example. The position of the germanium at the surface relative to the bulk can now be determined by the standing-wave method. For the (111) reflection case the standing-wave method measures only the distance $D$; it can give no information on the atomic arrangement in the plane. Thus the fluorescence yield for impurity atoms adsorbed at $A A^{\prime}$ or in the stacking-fault position $B B^{\prime}$ in the topmost layer would show the same angular

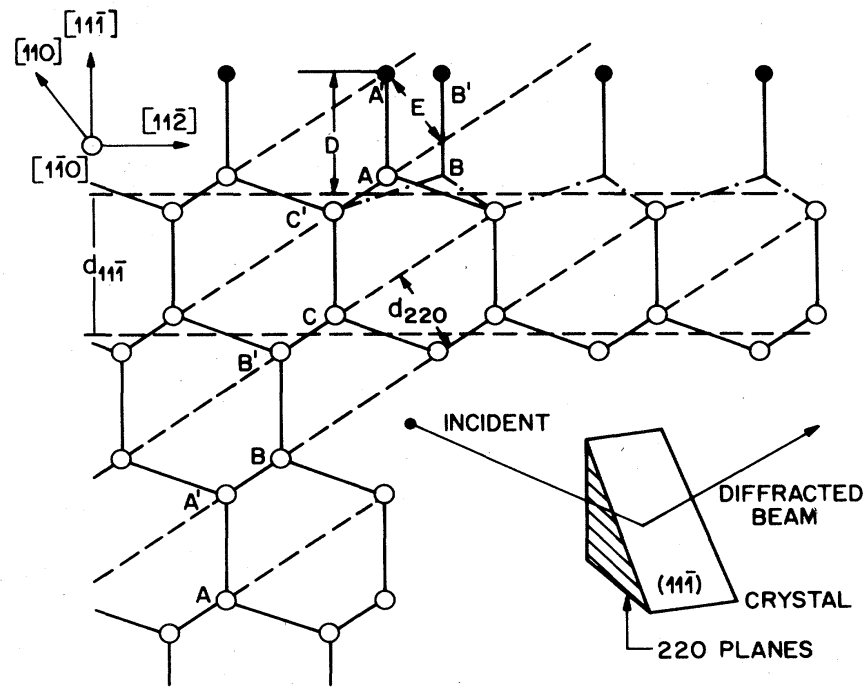

FIG. 2. Schematic of $\mathrm{Si}(111)$ surface viewed edge on along a [110] projection. Left half illustrates regular stacking $A A^{\prime} B B^{\prime} C C^{\prime}$, right half with stacking fault at the surface. Note positions of surface atoms (dark circles) in stacking-fault region with respect to (220) planes.

dependence in the Bragg band-gap region. If we, however, consider reflection from the (220) set of planes indicated by the dashed lines in Fig. 2, we see that the positions of the impurity atoms with respect to the (220) planes depend on whether the surface terminates at $A A^{\prime}$ bonds or in their counterpart in the stacking-fault configuration the bonds $B B^{\prime}$. The position of the impurity terminating the $B B^{\prime}$ bonds at the surface lies $\left(d_{220} / 3\right)$ below the $(220)$ planes. A theoretical calculation of the expected fluorescence for the faulted case is shown in Fig. 3 [curve (a)]. If such a surface displacement were present at reconstructed silicon surfaces it would be detected with ease by the method outlined above. The experimental results and theory are shown in curve (b) Fig. 3, and they clearly do not agree with the theoretical curve (a) for the stacking-fault model of the sur-

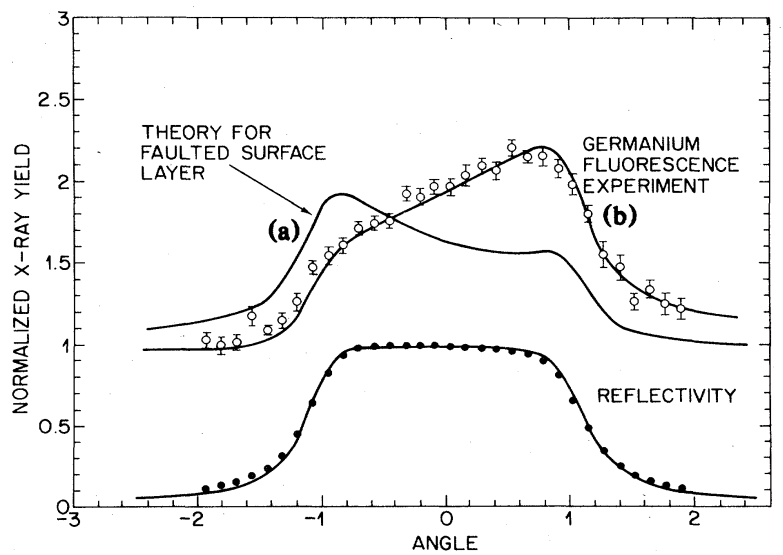

FIG. 3. Germanium fluorescence and reflectivity angular yields for (220) Bragg diffraction with a (111) surface. Theoretical yield curve for a faulted surface layer curve a has opposite symmetry to the experimental and theoretical result for an unfaulted surface curve $b$. 
face. ${ }^{13-15}$ They even have the wrong symmetry. A more appropriate model from our results in curve (b) places the coherent part of the signal directly on the (220) planes. The simple stacking-fault model is hence ruled out for our surface and, furthermore, the germanium is in registration with the silicon (220) planes as well as the (111) planes.

A clear picture of germanium atom positions thus emerges. We find that for coverages of and below $1 \mathrm{ML}$ germanium atoms occupy substitutional-like sites on both sublattice (111) planes at the surface. There is no simple stacking fault below these positions. In spite of previously mentioned claims that growth proceeds layer by layer ${ }^{6,7}$ our results clearly suggest that the fundamental process involves forming double layers of the diamond structure. This, of course, is not such an unrealistic suggestion since growth involving half the double layer has large numbers of unterminated bonds. Our result suggests that the conditions necessary for successful MBE growth on (111) surfaces may be just those that assure double-layer formation.

We now address the question of whether or not these results have any bearing on the original $(7 \times 7)$ silicon/germanium surface. Some caution is necessary here since it is not clear whether or not the amorphous silicon cap modifies the $(7 \times 7)$ structure at the interface. Firm evidence, that the $(7 \times 7)$ reconstruction is not modified by germanium deposited on silicon, was recently obtained by Gossman et al., ${ }^{16}$ who observe no modification of the characteristic $(7 \times 7)$ LEED pattern for various thicknesses of germanium up to $1000 \AA$ deposited on a $\mathrm{Si}(111)(7 \times 7)$ surface under experimental conditions identical to ours. On the other hand, preliminary transmission electron-diffraction experiments ${ }^{17}$ have failed to show the characteristic $(7 \times 7)$ structure on surfaces capped with $100 \AA$ of amorphous silicon. A further question concerns the effect of the amorphous layer on the proposed surface stacking fault. If the fault were to lie below the surface, as in early proposals, ${ }^{14}$ it is hard to envision its elimination by the capping procedure. However, more recent proposals suggest the fault lies at the surface, ${ }^{18}$ in which case it is hard to anticipate the influence of capping. Of course, it should be clear that the capping would be unnecessary for standing-wave experiments per- formed in UHV environments where such questions can be unambiguously resolved. We urge that $\mathrm{x}$-ray synchrotron facilities be committed to these kind of studies so that intensity problems associated with the UHV approach can be alleviated.

In view of the fact that at several points in our study we have called up channeling-backscattering methods for guidance (i.e., silicon surface peaks, coverages, amorphous nature of capped layer), it is interesting to discuss why, in view of the rather straightforward results obtained by standing waves for germanium submonolayer positions, these ion beam methods have as yet not yielded analogous information for this case. The $\mathrm{x}$-ray standing-wave measurements deduce atomic positions by locating the phase of standing waves induced by low-order Fourier components of the crystal charge density. As such, high-order Fourier components in the impurity density distribution do not play an inordinate role in the resulting fluorescence data. In channeling studies, the shadowing of lattice atoms by overlayer atoms shows an extreme sensitivity to high-order Fourier components of the density distribution, since the geometrical shadow cone is quite narrow requiring accurate atomic alignment. Effects causing a diminution of the high-order Fourier components (i.e., thermal vibrations, inhomogeneous strain fields, and/or static displacements) hence tend to reduce and mask lattice-location information. It should not be surprising that $\mathrm{x}$-ray standing-wave studies can yield useful results where channeling studies suggest only disorder at the surface.

Finally, we wish to emphasize that the standing-wave method has here successfully studied atomic structure at a buried interface below the amorphous cap. While the correspondence between this and UHV surfaces remains to be established we emphasize that such interfaces and impurities that reside there are of intrinsic interest in themselves and could well have significance in both fundamental and technological application.

We are indebted to P. E. Freeland and R. T. Lynch for their able assistance in various phases of this work.
"Present address: Department of Physics, Columbia University, New York, NY 10027.

1J. J. Lander, in Progress in Solid State Chemistry 2, edited by H. Reiss (Pergamon, New York, 1965), p. 26; Surf. Sci. 124, 106 (1980), and references therein.

${ }^{2}$ R. J. Culbertson, L. C. Feldman, and P. J. Silverman, Phys. Rev. Lett. 45, 2043 (1980); R. M. Tromp, E. J. Van Loenen, M. Larami, and F. W. Saris, Solid State Commun. 44, 971 (1981).

${ }^{3}$ K. Takayanagi, Y. Tanishiro, M. Takahashi, H. Motoyoshi, and $\mathrm{K}$. Yagi, in Proceedings of the Tenth Congress on Electron Microscopy, Hamburg, 1982 (unpublished), Vol. 2, p. 285; P. M. Petroff and R. J. Wilson, Phys. Rev. Lett. 51, 199 (1983).

${ }^{4}$ G. Binnig, H. Rohrer, G. H. Gerber, and E. Weibel, Phys. Rev. Lett. 50, 120 (1983).

${ }^{5}$ M. J. Cardillo, Phys. Rev. B 23, 4279 (1981).

${ }^{6}$ T. Narusawa and W. M. Gibson, Phys. Rev. Lett. 47, 1459 (1981).

${ }^{7}$ A. Shoji, J. Hyodo, H. Ueba, and C. Tatsuyama, Jpn. J. Appl. Phys. 22, 1482 (1983).
${ }^{8}$ H. J. Gossmann, L. C. Feldman, and W. M. Gibson, Phys. Rev. Lett. 53, 294 (1984); see also, Surf. Sci. (to be published).

9J. A. Golovchenko, J. R. Patel, D. R. Kaplan, P. L. Cowan, and M. J. Bedzyk, Phys. Rev. Lett. 49, 560 (1982).

10J. R. Patel and J. A. Golovchenko, Phys. Rev. Lett. 50, 1858 (1983).

11 J. C. Bean and E. A. Sadowski, J. Vac. Sci. Technol. 20, 137 (1982).

${ }^{12} \mathrm{We}$ are grateful to L. C. Feldman for assistance with these experiments and interpretation.

${ }^{13}$ P. M. Petroff and R. J. Wilson, Phys. Rev. Lett. 51, 199 (1983).

${ }^{14}$ E. G. McRae, Phys. Rev. B 28, 2305 (1983).

${ }^{15}$ P. A. Bennett, L. C. Feldman, Y. Kuk, E. G. McRae, and J. E. Rowe, Phys. Rev. B 28, 3656 (1983).

${ }^{16}$ H. J. Gossmann, J. C. Bean, L. C. Feldman, E. G. McRae, and I. K. Robinson, J. Vac. Sci. Technol. (to be published).

${ }^{17} \mathrm{P}$. M. Petroff (private communication).

${ }^{18}$ K. Takayanagi, K. Yagi, Y. Tanishiro, and K. Kobayashi, J. Vac. Sci. Technol. (to be published). 